MATEC Web of Conferences 6, 03006 (2013)

DOI: $10.1051 /$ matecconf/20130603006

(C) Owned by the authors, published by EDP Sciences, 2013

\title{
Measurement systems to detect the time-dependant development of concrete spalling under fire exposure
}

\author{
R. Richter ${ }^{1}$, F. Dehn ${ }^{2}$, J. Schmidt ${ }^{2}$, M. Juknat ${ }^{2}$ and C.U. Grosse ${ }^{1}$ \\ ${ }^{1}$ Chair of Non-destructive Testing, Technical University of Munich, Germany \\ 2 Leipzig Institute for Materials Research and Testing (MFPA), Leipzig, Germany
}

\begin{abstract}
In order to analyse the behaviour of concrete under fire exposure experimental investigations are used [1]. This practice is expensive and very time-consuming. So, it is essential to get as most suitable information out of such experiments as possible. At the present, most standard experiments are only capable of comparing a condition of concrete before and after the experiments [1]. Unfortunately, information of the time-dependent development of the damaging process cannot be resolved. In order to fully understand the mechanism of dynamic concrete damage under fire exposure, this information is crucial. Therefore, it is necessary to develop measurement systems which are able to monitor and detect the entire exposure process and its consequences. These systems should be applied additionally to the temperature monitoring, the visual inspection and a weighting of the specimen before and after the experiments. For this purpose volume-oriented non-destructive testing methods are considered. It is possible to use ultrasonic systems or impact-echo methods, but the most suitable are acoustic emission techniques. With this method it is possible to monitor damage processes of the complete specimen volume and during the whole time of the experiments. The paper will present results of experimental investigations where such systems have been successfully applied to test the feasibility, practicability and the usefulness of different measurement systems to verify the time-dependant development of concrete spalling under fire exposure.
\end{abstract}

\section{INTRODUCTION}

Acoustic emission analysis (AE) is a passive non-destructive testing method (ndt). A damage process like a crack inside the material generates a burst-signal. Starting from the source, an elastic wave propagates through the specimen. Similar to the earthquake detection in seismology these burst signals can be detected and recorded with sensors on the surface. An analysis of these signals enables conclusions about the damage processes. So it is a volume-oriented measurement system which is able to monitor the time-development of concrete damage. It was already used for different applications in civil-engineering research [2]. Preliminary experiments could show that it is possible to use acoustic emission techniques to support investigations on concrete under fire exposure $[3,5]$.

For the practical use of acoustic emission analysis some boundary conditions must be considered. It is necessary to choose suitable sensor-types, the coupling of the sensors to the surface, the sensorarrangement and the setup of preamplifiers, hardware filter and transient recorders. Furthermore, difficulties because of noise, for example generated by the oil-burners, should be considered.

\section{SETUP AND EQUIPMENT}

For the application of the acoustic emission analysis at least one side of the specimen surface must be accessible. The working temperature of the most AE-sensors is specified at about $60^{\circ} \mathrm{C}$. Coaxial cables usually have no temperature protection. These difficulties can be solved by the use of an experimental

This is an Open Access article distributed under the terms of the Creative Commons Attribution License 2.0, which permits unrestricted use, distribution, and reproduction in any medium, provided the original work is properly cited. 


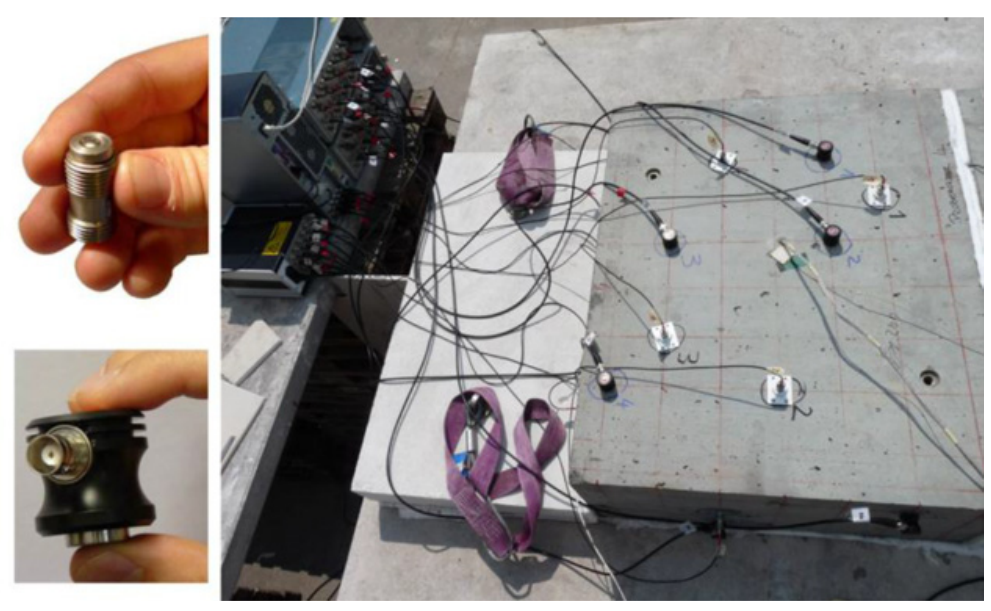

Figure 1. Different types of sensors and their application on the surface of the specimen.

setup with a fire exposure from one side. On the other side, the sensors can be installed [Fig. 1]. The time, the temperature needs to go through the concrete can be used for the measurement. At a critical surface temperature the sensors must be removed.

After some investigations of coupling methods, hot glue shows the best feasibility to install the sensors. The preparation is very fast, the sensors need no other bracket, the signal transmission is very good and it is easy to remove the sensors if necessary. The sensors should be arranged over the whole surface to get a senor distribution as evenly as possible. To prevent geometrical effects concerning localization an unsymmetrical sensor distribution is required. Different types of sensors have been tested [Fig. 1]. For a signal based acoustic emission analysis it is important to use broadband sensors in the right frequency range of AE events in concrete. Furthermore, the sensors have to be easy and fast installed and must be rugged enough.

Pre-amplifiers are in use to increase the signal amplitude. This is very important to detect low signals and to resolve for example micro-cracking-effects. The amplitude of signals due to spalling effect is bigger. So for every application a suitable measurement setup must be determined. To avoid problems with the data-collection because of noise the analog signals should be filtered. The measurement system records on 16 Channels with a sampling rate of $1 \mathrm{MHz}$ which is sufficient to sample the important frequency ranges of $\mathrm{AE}$ events in concrete.

In the experimental program, concrete with and without polypropylene (PP) fibres were tested. The oil-burner-furnace at the MFPA Leipzig were controlled after the ISO 834 and the modified ZTV-ING temperature curves [Fig. 2 right]. In every experiment specimens with a size of $70 \times 85 \times 30 \mathrm{~cm}^{3}$ have been used. Figure 2, left, show the specimen on top of the surface and Figure 3 the damaged specimens after the experiment. In the concrete mix with PP-fibres, no spalling occurred. On every specimen bending cracks were observed.

Additional to the AE-measurement systems, a temperature monitoring device inside the specimens and the furnace chamber recorded information about the temperature development during the experiments.

\section{RESULTS}

\subsection{Acoustic emission activity}

To get information about the time-dependent damage behaviour of the material, a first opportunity is to plot the cumulative frequency of events against the time. The gradient of this acoustic emission activity 

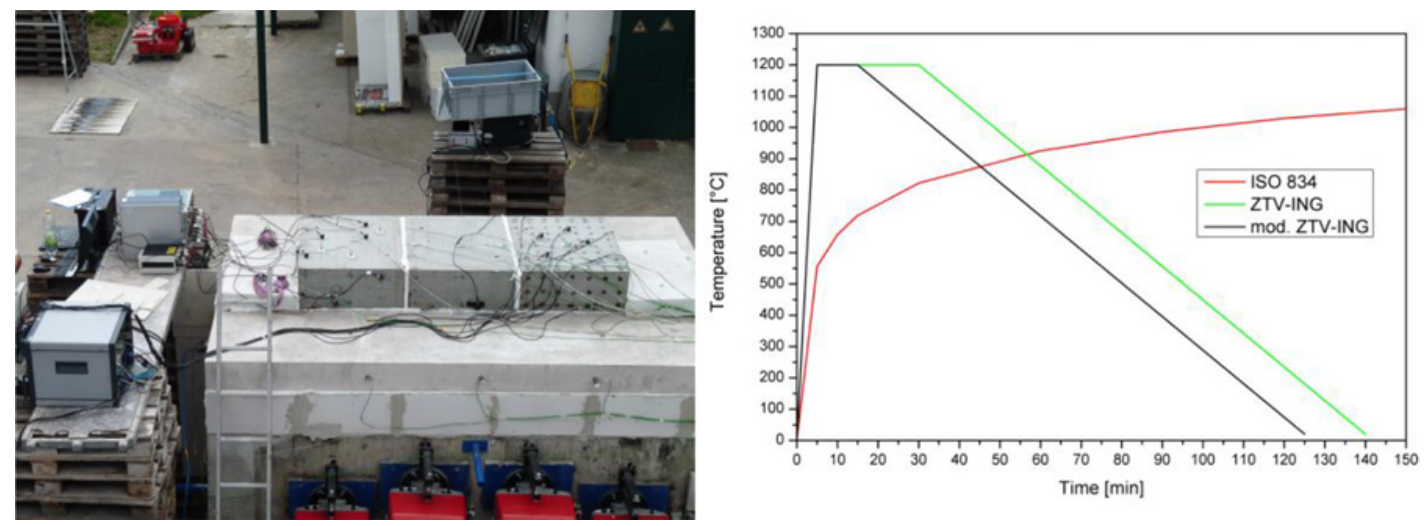

Figure 2. Specimens on top of the furnace with the connected measurement systems (left) and the used temperatecurves (right). The specimens were tested according to the ISO 834 and the modified ZTV-ING.

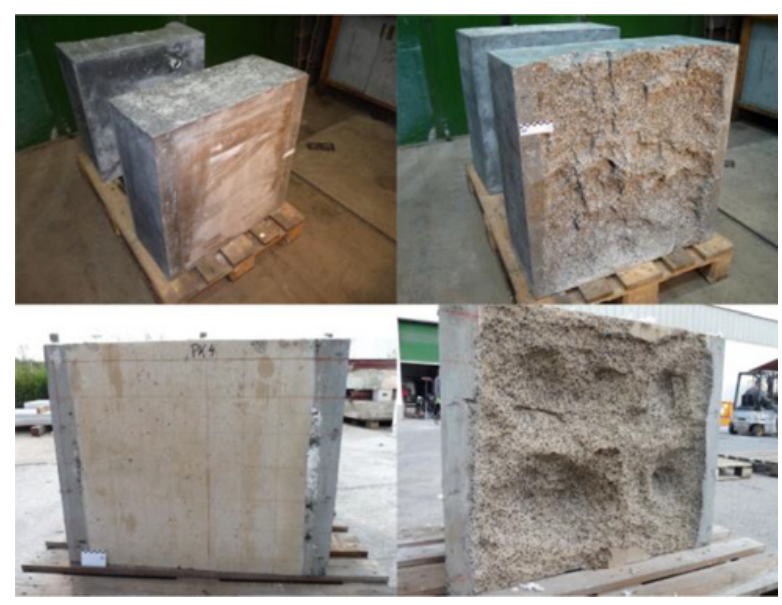

Figure 3. Specimen with $1 \mathrm{~kg}$ PP fibres per $\mathrm{m}^{3}$ concrete after the experiment (left), specimen without PP fibres showing damage due to spalling (right).

delivers a relation with increased occurance of damage processes in the concrete. A comparison between a specimen with and without PP fibers can be found in Figure 4, left. The gradient and the total number of occurring events of the AE activity without fibers are much higher. One can assume that these different results had been generated by spalling events. Events obtained in the specimen with PP fibres, where no spalling occurred [Fig. 3, left] are caused by orthogonal bending cracks because of temperature stresses, micro cracking and noise of the fire testing equipment. All these influences generated AE events which are recorded by the measurement system and can therefore be found in the AE results.

Figure 4, right, shows the difference in the acoustic emission activity generated by the use of two different temperature curves [Fig. 2, right]. The difference in the gradient in the beginning and the limit after ca. 10 min by the use of the modified ZTV-ING curve corresponds to the temperature exposure.

The acoustic emission activity is a useful tool to get an overview about a measurement and a possibility for a fast and easy comparison between different experiments. Furthermore, it is an understandable and clear illustration, including the time information, and delivers a two dimensional plot which can be compared with other measurement results. 


\section{MATEC Web of Conferences}
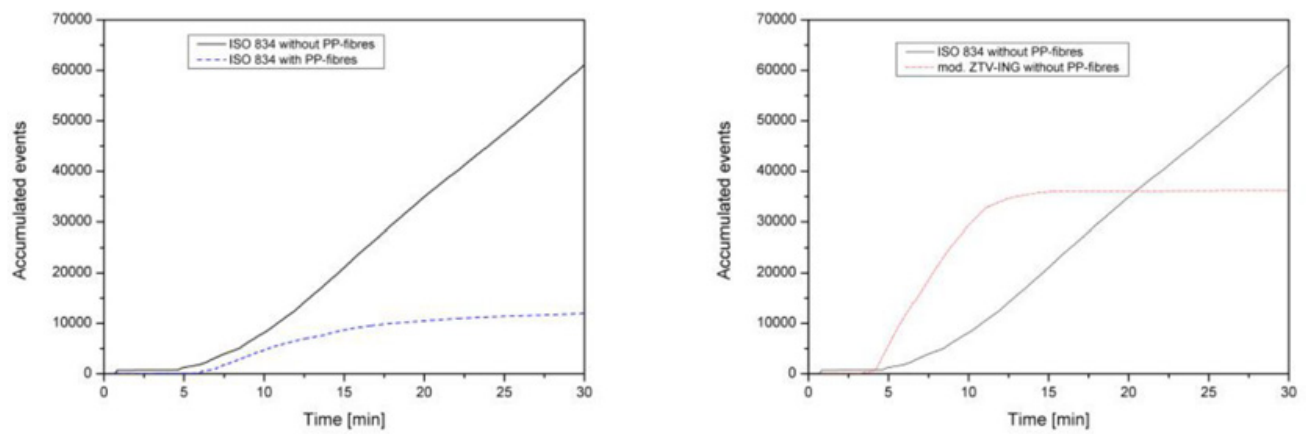

Figure 4. Acoustic emission activity of specimen with and without PP-fibres tested after the modified ZTV-ING (left) and the comparison of the acoustic emission activity from experiments with different temperature curves (right).
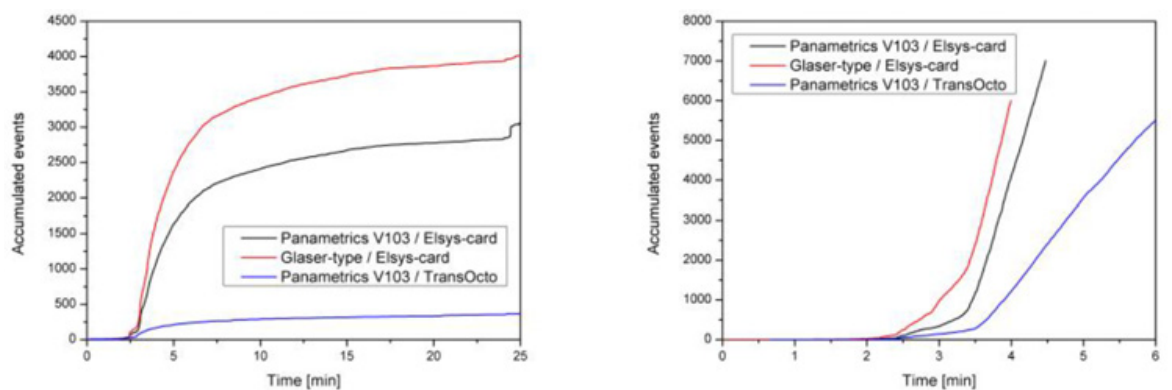

Figure 5. The difference of the acoustic emission activity by the use of different measurement systems and sensor types. Left, specimen with PP-fibres, right without PP-fibres.

Additional to the mentioned difficulties that the AE results includes events because of cracking, spalling, micro cracking and noise, there are some other effects which influence the AE activity. An example is the specimen size. The AE analysis detects events over the whole specimen. By the use of bigger specimen, the surface under fire exposure is higher, so in total the number of $\mathrm{AE}$ events will increase although the same material is tested. Another influence is the measurement system and the measurement settings. Each type of sensor and each type of preamplifier are different in sensitivity and possibilities for adjustment. Especially the adjustment of the trigger conditions is critical for the acoustic emission activity. Figure 5 shows the AE activity of two experiments (one with and one without PP fibres) by the use of three different measurement systems. The deviations are significant, so the usability must be questioned. The agreement between the three curves is recognizable, so it is principle possible to get information out of these plots. Information like the beginning or time ranges with high damage activity can be careful estimated. But as one can see in Figure 5 left between approx. for 2 min and $4 \mathrm{~min}$, the curve profile can be very different. Thus it can be a problem to close on material behavior out of these results. To achieve this further evaluation methods are required.

\subsection{Discrimination between cracking and spalling on base of the signal energy}

For an investigation of spalling effects it is important to discriminate spalling and delamination parallel to the surface from orthogonal bending cracks, micro cracking and other effects. A possibility to get more parameters in the plots is a representation of the signal-energy over the time. Every point represents 

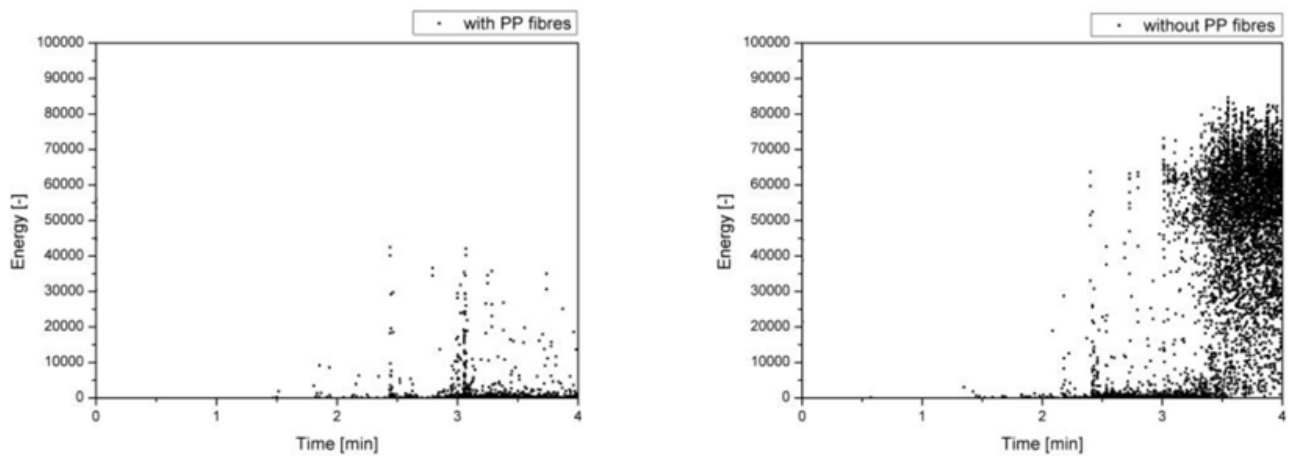

Figure 6. Signal energy of the AE events over the time. Comparison, between experiments with (left) and without (right) PP fibres.

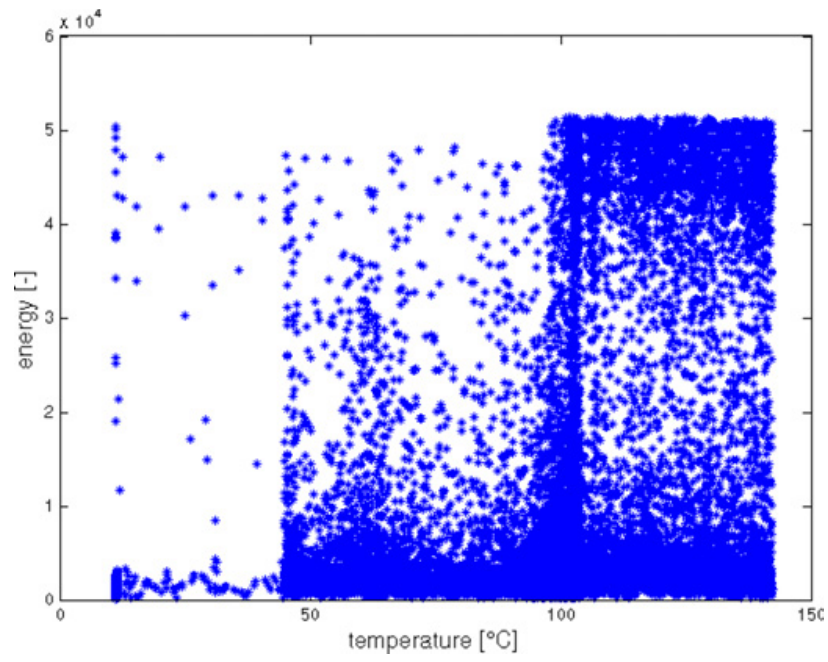

Figure 7. Occurrence of AE events and their signal-energy plotted over temperature.

an AE event, so the acoustic emission activity can be achieved as a density of points in an area of the plot.

Looking at an experiment with and one without PP-fibres the difference can be seen in the events with high energy. By using PP fibres, no spalling but cracking could be observed [Fig. 3, left]. It seems that it is possible to discriminate spalling effects on the basis of this method. So signals of lower energy would represent micro cracking and bending cracks. This corresponds with the obtained results because the pattern in this area seems to be mostly equal in the two experiments.

\subsection{Relation between temperature exposure and the signal energy}

Based on the hypothesis that the signal energy includes information about the type of damage, it was connected with the results of the temperature measurement. The temperature curve from one temperature measurement point inside the specimen, in $2 \mathrm{~cm}$ depth from surface, was used.

For a specimen without PP fibres the result is shown in Figure 7. Three significant sections are recognizable. The beginning of a high activity in a lower energy level can be seen at approx. $45^{\circ} \mathrm{C}$ and the increasing occurrence of high energy-signals at $100^{\circ} \mathrm{C}$. Assuming that signals with a high energy 


\section{MATEC Web of Conferences}
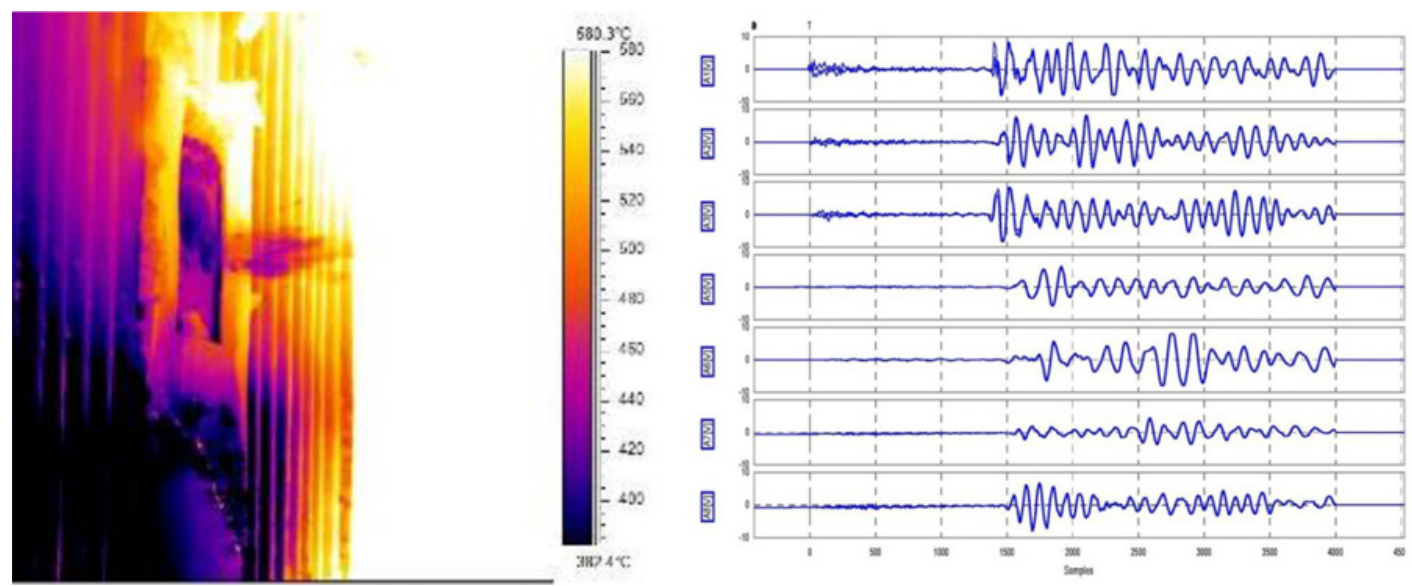

Figure 8. Infrared-thermography of a spalling event (left) and AE-signals, captured with an eight-channel transient recorder (right).

are linked with spalling processes the boundary at $100{ }^{\circ} \mathrm{C}$ could be an indication for pore-pressure as a cause of spalling.

\subsection{Comparison with video and infrared-thermography}

To verify these observations, it is important to compare the results with other information. One possibility is the use of a video-camcorder. However, the visual monitoring is often not possible. The view to the concrete surface inside the furnace is limited because of the size of the observation windows. Another difficulty for video-systems are flames and fire smoke.

This visual disability is no problem for infrared-thermography-cameras. But here, a free view to the surface is required. Additional to the time a spalling occurs it is possible to get information about the temperature-allocation of the surface and the surface after spalling. Figure 8 left shows such an infraredthermography picture of a spalling event. Synchronized with the AE-system it might be possible to identify single signals generated by spalling.

\section{CONCLUSIONS AND OUTLOOK}

The presented results show that it is possible to use acoustic-emission-analysis to monitor the timedependant behaviour of concrete under fire exposure. Different display options seem to be able to allow conclusions about the damage processes. Based on these results, the authors started a research project to verify the observations and to extend the analysis software for this application which is presently under investigation.

Additional to the presented possibilities of analysis, it is very important to calculate the localization of acoustic-emission-events. Furthermore to work with localization results statements about the accuracy of the localization is required. This is needed to identify real events out of the specimen against noise. And the three-dimensional location information could be used to detect the time-dependent depth of spalling. Difficulties for the calculation of localisations are the changing of the acoustic velocity in concrete, because of temperature exposure [4], the sensor arrangement and the sizes of the specimens. In a new research project these problems will now be handled. The intention is to improve the localization with the use of acoustic velocity models. 
To investigate the accuracy and the usability of the presented methods the research project includes an experimental program with different concrete mixtures, temperature curves and observations of other influences like humidity or compressive stress.

The authors are grateful for a research project (Feb. 2010 - Feb. 2012) sponsored by the German research foundation (Deutsche Forschungsgemeinschaft, DFG) via grant GR1664/7-1 and for the collaboration in this project with the Department of Construction Materials, University of Stuttgart, Germany. The new research project started in March 2013 and is funded by the German Federal Ministry of Economics and Technology (BMWi).

\section{References}

[1] Dehn F. et al.: Fire and spalling behaviour of fibre reinforced concrete in road tunnels, Report B73, Federal Highway Research Institute (BASt), 2010.

[2] Grosse C., Ohtsu M. (Eds.): Acoustic Emission Testing in Engineering - Basics and Applications. ISBN: 978-3-540-69895-1, 415 S., Springer publ., Heidelberg, 2008.

[3] Grosse C., Richter R., Ozbolt J., Dehn F., Juknat M.: Spalling of HPC evaluated by acoustic emission an numerical analysis. ${ }^{\text {nd }}$ International RILEM Workshop on Concrete Spalling due to Fire Exposure 5-7 October 2011, Delft, The Netherlands, 2011.

[4] Krapp M.: Development of the ultrasonic-velocity of high performance concrete under fire exposure. Master's thesis, Technical University of Munich, 2012 (in German).

[5] Richter R: Acoustic emission analysis to detect the cracking and spalling behavior of concrete under fire exposure. Diploma thesis, MPA University Stuttgart, 2009 (in German). 